\title{
BOUNDARY VALUE PROBLEMS FOR THE STATIONARY NORDSTRÖM-VLASOV SYSTEM
}

\author{
Mihai Bostan
}

\begin{abstract}
We study the existence of weak solution for the stationary Nordström-Vlasov equations in a bounded domain. The proof follows by fixed point method. The asymptotic behavior for large light speed is analyzed as well. We justify the convergence towards the stationary Vlasov-Poisson model for stellar dynamics.
\end{abstract}

\section{Introduction}

We consider a population of particles and we assume that collisions are so rare such that we can neglect them. In plasma physics the charged particles interact by electro-magnetic forces and the evolution of the system is described by the Vlasov-Maxwell equations. When the magnetic field is negligible we can use the Vlasov-Poisson system. In astrophysics large stellar systems such as galaxies or globular clusters interact by gravitational forces and the dynamics of the system is given by the Einstein-Vlasov equations.

The Cauchy problem for the Vlasov-Poisson and Vlasov-Maxwell systems are now well understood, cf. [3, 26, 29, 33, 35, 39], respectively $[10,16,19$, $20,21,24]$. There are also some results for initial-boundary value problems $[1,23]$ and boundary value problems $[7,22,30,31]$. A lot of studies concerns the stationary solutions and their stability $[5,6,18,32,34]$.

It is well known that the Vlasov-Poisson model can be derived from the relativistic Vlasov-Maxwell model assuming that the particle velocities are small compared to the light speed $[8,15,25,38]$.

The Einstein-Vlasov system is much more difficult, see $[2,36,37]$. A simplified relativistic model is obtained by coupling the Vlasov equation to the Nordström scalar gravitation theory [28].

We denote by $\digamma=\digamma(t, x, p) \geq 0$ the particle density in the phase-space. Here $t \in \mathbb{R}$ represents the time, $x \in \mathbb{R}^{N}$ the position and $p \in \mathbb{R}^{N}$ the impulsion,

Received July 1, 2008; Revised June 5, 2009.

2000 Mathematics Subject Classification. 35B35, 35A05, 35B45.

Key words and phrases. Nordström equation, Vlasov equation, Poisson equation, weak/ mild solutions. 
with $N \geq 1$. The density $\digamma$ satisfies the following kinetic equation

$$
\partial_{t} \digamma+v_{c}(p) \cdot \nabla_{x} \digamma-\left(\left(\partial_{t} \phi+v_{c}(p) \cdot \nabla_{x} \phi\right) \frac{p}{m c^{2}}+\frac{\nabla_{x} \phi}{\gamma_{c}(p)}\right) \cdot \nabla_{p} \digamma=0,
$$

coupled to the wave equation

$$
\frac{1}{c^{2}} \partial_{t}^{2} \phi-\Delta_{x} \phi=-e^{\frac{N+1}{m c^{2}} \phi(t, x)} \int_{\mathbb{R}^{N}} \frac{\digamma(t, x, p)}{\gamma_{c}(p)} d p .
$$

Here $m$ is the mass of particles, $c$ is the light speed in the vacuum, $\gamma_{c}(p)=$ $\left(1+\frac{|p|^{2}}{m^{2} c^{2}}\right)^{\frac{1}{2}}, p \in \mathbb{R}^{N}$ is the Lorentz factor and $v_{c}(p)=\frac{p}{m \gamma_{c}(p)}$ is the relativistic velocity of a particle with impulsion $p \in \mathbb{R}^{N}$. For more details on the model see [11]. After introducing the new unknown $f(t, x, p)=e^{\frac{N+1}{m c^{2}} \phi(t, x)} \digamma(t, x, p)$ the system becomes

$$
\partial_{t} f+v_{c}(p) \cdot \nabla_{x} f+F(t, x, p) \cdot \nabla_{p} f=\frac{N+1}{m c^{2}} f(t, x, p) S \phi
$$

$$
\frac{1}{c^{2}} \partial_{t}^{2} \phi-\Delta_{x} \phi=-\mu(t, x)
$$

$$
\mu(t, x)=\int_{\mathbb{R}^{N}} \frac{f(t, x, p)}{\gamma_{c}(p)} d p
$$

where $S=\partial_{t}+v_{c}(p) \cdot \nabla_{x}$ is the free-transport operator and $F(t, x, p)=$ $-\left(S \phi \frac{p}{m c^{2}}+\frac{\nabla_{x} \phi}{\gamma_{c}(p)}\right)$. We impose the initial conditions

$$
f(0, \cdot, \cdot)=f_{0}, \quad \phi(0, \cdot)=\varphi_{0}, \partial_{t} \phi(0, \cdot)=\varphi_{1} .
$$

The Nordström-Vlasov system (3), (4), (5), (6) was analyzed recently by Calogero and Rein. They proved that classical solutions exist at least locally in time in three dimensions and globally in time in one dimension, cf. [13]. The existence of global weak solutions is obtained in [14]. The convergence towards the gravitational Vlasov-Poisson model when the light speed becomes large is justified in [12].

The aim of this paper is to construct weak solutions for the stationary boundary value Nordström-Vlasov system

$$
\begin{aligned}
& v_{c}(p) \cdot \nabla_{x} f-\left(v_{c}(p) \cdot \nabla_{x} \phi \frac{p}{m c^{2}}+\frac{\nabla_{x} \phi}{\gamma_{c}(p)}\right) \cdot \nabla_{p} f \\
= & \frac{N+1}{m c^{2}} f(x, p) v_{c}(p) \cdot \nabla_{x} \phi, \quad(x, p) \in \Omega \times \mathbb{R}^{N},
\end{aligned}
$$

$$
-\Delta_{x} \phi=-\mu(x), \quad x \in \Omega,
$$

$$
\mu(x)=\int_{\mathbb{R}^{N}} \frac{f(x, p)}{\gamma_{c}(p)} d p, \quad x \in \Omega,
$$


with the boundary conditions

$$
f(x, p)=g(x, p), \quad(x, p) \in \Sigma^{-}, \quad \phi(x)=\varphi_{0}(x), \quad x \in \partial \Omega,
$$

with $N \geq 2$. For the one dimensional case the reader can refer to [9]. Here $\Omega$ is a smooth open bounded set of $\mathbb{R}^{N}, \Sigma^{-}=\left\{(x, p) \in \partial \Omega \times \mathbb{R}^{N}: p \cdot n(x)\right.$ $<0\}$, where $n(x)$ represents the unit outward normal to $\partial \Omega$ at $x$ and $g, \varphi_{0}$ are given functions. We introduce also the notations $\Sigma=\partial \Omega \times \mathbb{R}^{N}, \Sigma^{+}=\{(x, p) \in$ $\left.\partial \Omega \times \mathbb{R}^{N}: p \cdot n(x)>0\right\}$, we denote by $d \sigma$ the superficial measure on $\partial \Omega$ and we consider the measures $d \nu^{ \pm}$on $\Sigma^{ \pm}$given by $d \nu^{ \pm}=\left|v_{c}(p) \cdot n(x)\right| d \sigma(x) d p$. By weak solution for the stationary boundary value Nordström-Vlasov system (7), $(8),(9),(10)$ we understand a couple $(f, \phi)$ such that $f$ satisfies the stationary Vlasov equation (7) with the boundary condition $f(x, p)=g(x, p), \quad(x, p) \in \Sigma^{-}$ in the sense of distributions (see Section 2 for exact definitions and main properties) and $\phi$ is a weak solution of the Poisson equation (8) with the boundary condition $\phi(x)=\varphi_{0}(x), \quad x \in \partial \Omega$. Basically we assume that the inflow boundary condition $g$ is non negative, bounded, locally integrable on $\Sigma^{-}$such that $\int_{p \cdot n(\cdot)<0} \frac{g(\cdot, p)}{\gamma_{c}(p)} d p \in L^{\infty}(\partial \Omega)$. In particular we establish our results for inflow boundary conditions $g$ satisfying $0 \leq g(x, p) \leq C(1+|p|)^{-\delta},(x, p) \in \Sigma^{-}$. Clearly such boundary conditions belong to $L_{\text {loc }}^{1} \cap L^{\infty}\left(\Sigma^{-} ; d \nu^{-}\right)$and satisfy $\int_{p \cdot n(\cdot)<0} \frac{g(\cdot, p)}{\gamma_{c}(p)} d p \in L^{\infty}(\partial \Omega)$ for any $\delta>N-1$. One of the key points is to take advantage of the conservation of the particle total energy along characteristics. We use the method introduced by Poupaud in [30], but in a gravitational case. We obtain the following existence result

Theorem 1.1. Assume that $\varphi_{0} \in H^{1 / 2}(\partial \Omega) \cap L^{\infty}(\partial \Omega), \varphi_{0} \geq 0$ on $\partial \Omega, g \geq 0$ on $\Sigma^{-}$such that

$$
g(x, p) \leq \frac{C}{(1+|p|)^{\delta}}, \quad(x, p) \in \Sigma^{-}
$$

for some constants $C>0, \delta>N-1, N \geq 2$. Then, for any $c>0$ there is a weak solution $\left(f_{c}, \phi_{c}\right)$ for the stationary Nordström-Vlasov system (7), (8), (9), (10) satisfying

$$
\begin{gathered}
0 \leq f_{c} \leq e^{\frac{N+1}{m c^{2}}\left\|\varphi_{0}\right\|_{L^{\infty}(\partial \Omega)}}\|g\|_{L^{\infty}\left(\Sigma^{-}\right)}, \int_{\mathbb{R}^{N}} \frac{f_{c}(\cdot, p)}{\gamma_{c}(p)} d p \in L^{\infty}(\Omega), \\
\phi_{c} \in H^{1}(\Omega), \lim _{R \rightarrow+\infty}\left\|\int_{|p| \geq R} \frac{f_{c}(\cdot, p)}{\gamma_{c}(p)} d p\right\|_{L^{\infty}(\Omega)}=0 .
\end{gathered}
$$

Moreover, if $\delta>2 N, N \in\{2,3\}$ we have

$$
\sup _{c \geq 1}\left\|\int_{\mathbb{R}^{N}} f_{c}(\cdot, p) d p\right\|_{L^{\infty}(\Omega)}<+\infty, \lim _{R \rightarrow+\infty} \sup _{c \geq 1}\left\|\int_{|p| \geq R} f_{c}(\cdot, p) d p\right\|_{L^{\infty}(\Omega)}=0 .
$$

We justify also the asymptotic behavior towards the stationary boundary value Vlasov-Poisson system for large light speed. As before, by weak solution 
for the stationary boundary value Vlasov-Poisson system we understand a couple $(f, \phi)$ such that $f$ satisfies the Vlasov problem in the sense of distributions and $\phi$ is a weak solution for the Poisson problem.

Theorem 1.2. Assume that $\varphi_{0} \in H^{1 / 2}(\partial \Omega) \cap L^{\infty}(\partial \Omega), \varphi_{0} \geq 0$ on $\partial \Omega, g \geq 0$ on $\Sigma^{-}$such that

$$
g(x, p) \leq \frac{C}{(1+|p|)^{\delta}}, \quad(x, p) \in \Sigma^{-}
$$

for some constants $C>0, \delta>2 N, N \in\{2,3\}$. For any $c \geq 1$ let $\left(f_{c}, \phi_{c}\right)$ be the weak solution of the stationary Nordström-Vlasov system constructed in Theorem 1.1. Then there is a sequence $\left(c_{k}\right)_{k}, \lim _{k \rightarrow+\infty} c_{k}=+\infty$ such that

$$
\begin{gathered}
f_{k}:=f_{c_{k}} \rightarrow f, \text { weakly } \star \text { in } L^{\infty}\left(\Omega \times \mathbb{R}^{N}\right), \\
\phi_{k}:=\phi_{c_{k}} \rightarrow \phi, \text { strongly in } H^{1}(\Omega),
\end{gathered}
$$

where $(f, \phi)$ is a weak solution of the Vlasov-Poisson system

$$
\begin{gathered}
\frac{p}{m} \cdot \nabla_{x} f-\nabla_{x} \phi \cdot \nabla_{p} f=0, \quad(x, p) \in \Omega \times \mathbb{R}^{N}, \\
-\Delta_{x} \phi=-\rho(x):=-\int_{\mathbb{R}^{N}} f(x, p) d p, \quad x \in \Omega, \\
f(x, p)=g(x, p), \quad(x, p) \in \Sigma^{-}, \quad \phi(x)=\varphi_{0}(x), \quad x \in \partial \Omega .
\end{gathered}
$$

Our paper is organized as follows. In Section 2 we recall the notions of weak and mild solutions for the stationary Vlasov problem and we present the properties of such solutions. In particular we deduce estimates for the mild solution, some of them being independent of the light speed. In Section 3 we construct a fixed point map for a regularized Nordström-Vlasov system and we show the existence of a fixed point by using the Schauder theorem. The existence of weak solution for the Nordström-Vlasov system is obtained in Section 4 by weak stability. We prove also the convergence towards the gravitational Vlasov-Poisson system when the light speed goes to infinity.

\section{The Vlasov equation}

In this paragraph we assume that $\phi=\phi(x), g=g(x, p)$ are given functions and we introduce the notions of weak and mild solutions for the stationary Vlasov problem

$$
\begin{gathered}
v_{c}(p) \cdot \nabla_{x} f+F(x, p) \cdot \nabla_{p} f=\frac{N+1}{m c^{2}} f(x, p) S \phi, \quad(x, p) \in \Omega \times \mathbb{R}^{N} \\
f(x, p)=g(x, p), \quad(x, p) \in \Sigma^{-}
\end{gathered}
$$


where $S=v_{c}(p) \cdot \nabla_{x}$ and $F(x, p)=-\left(S \phi \frac{p}{m c^{2}}+\frac{\nabla_{x} \phi}{\gamma_{c}(p)}\right)$. Observe that the divergence of the field $\left(v_{c}(p), F(x, p)\right)$ with respect to the variables $(x, p)$ is given by

$$
\operatorname{div}_{(x, p)}\left(v_{c}(p), F(x, p)\right)=-\frac{N}{m c^{2}} S \phi
$$

Therefore the Vlasov equation (14) can be written formally $\operatorname{div}_{x}\left(v_{c}(p) f(x, p) e^{-\frac{\phi(x)}{m c^{2}}}\right)+\operatorname{div}_{p}\left(F(x, p) f(x, p) e^{-\frac{\phi(x)}{m c^{2}}}\right)=0, \quad(x, p) \in \Omega \times \mathbb{R}^{N}$. We have the usual definition for the weak solution:

Definition 2.1. Assume that $\phi \in W^{1, \infty}(\Omega), g \in L_{\text {loc }}^{1}\left(\Sigma^{-} ; d \nu^{-}\right)$. We say that $f \in L_{\text {loc }}^{1}\left(\bar{\Omega} \times \mathbb{R}^{N}\right)$ is a weak solution for the stationary Vlasov problem (14), (15) if and only if

$$
\begin{aligned}
& -\int_{\Omega} \int_{\mathbb{R}^{N}} f(x, p) e^{-\frac{\phi(x)}{m c^{2}}}\left(v_{c}(p) \cdot \nabla_{x} \theta+F(x, p) \cdot \nabla_{p} \theta\right) d p d x \\
= & \int_{\Sigma^{-}} g(x, p) e^{-\frac{\phi(x)}{m c^{2}}} \theta d \nu^{-}
\end{aligned}
$$

for any test function $\theta \in C_{c}^{1}\left(\bar{\Omega} \times \mathbb{R}^{N}\right)$ satisfying $\left.\theta\right|_{\Sigma^{+}}=0$.

Assume now that $\phi \in W^{2, \infty}(\Omega)$ and for any $(x, p) \in\left(\Omega \times \mathbb{R}^{N}\right) \cup \Sigma^{-}$let us introduce the system of characteristics

$$
\frac{d X}{d s}=v_{c}(P(s)), \quad \frac{d P}{d s}=F(X(s), P(s)),
$$

with the conditions

$$
X(s=0)=x, P(s=0)=p .
$$

Notice that under the above regularity hypothesis on $\phi$ there is a unique $C^{1}$ solution $(X(s), P(s)):=(X(s ; x, p), P(s ; x, p))$ of $(18),(19)$ for $s \in] s_{\text {in }}(x, p)$, $s_{\text {out }}(x, p)[$, where the entry/exit times are given by

$$
\begin{gathered}
s_{\text {in }}(x, p)=\inf \{\tau \leq 0: X(s ; x, p) \in \Omega, \forall s \in] \tau, 0[\}, \\
s_{\text {out }}(x, p)=\sup \{\tau \geq 0: X(s ; x, p) \in \Omega, \forall s \in] 0, \tau[\} .
\end{gathered}
$$

Observe that the Vlasov equation (14) can be written

$$
v_{c}(p) \cdot \nabla_{x}\left(f(x, p) e^{-\frac{N+1}{m c^{2}} \phi(x)}\right)+F(x, p) \cdot \nabla_{p}\left(f(x, p) e^{-\frac{N+1}{m c^{2}} \phi(x)}\right)=0,(x, p) \in \Omega \times \mathbb{R}^{N},
$$

saying that $f(x, p) e^{-\frac{N+1}{m c^{2}} \phi(x)}$ is constant along all characteristics. We have the definition:

Definition 2.2. Assume that $\phi \in W^{2, \infty}(\Omega)$. The mild solution (or solution by characteristics) of the stationary Vlasov problem (14), (15) is given by

$f(x, p)=e^{\frac{N+1}{m c^{2}} \phi(x)} e^{-\frac{N+1}{m c^{2}} \phi\left(X\left(s_{\text {in }} ; x, p\right)\right)} g\left(X\left(s_{\text {in }} ; x, p\right), P\left(s_{\text {in }} ; x, p\right)\right)$, if $s_{\text {in }}(x, p)>-\infty$, 
and

$$
f(x, p)=0 \text { if } s_{\text {in }}(x, p)=-\infty .
$$

By definition the mild solution is unique. Unfortunately, in general there is no uniqueness for the weak solution because $f$ can take arbitrary values on the characteristics such that $s_{\text {in }}=-\infty$. In order to retrieve the uniqueness of the weak solution we penalize the Vlasov equation. For any $\alpha>0$ we consider the problem

(22) $\alpha f(x, p)+v_{c}(p) \cdot \nabla_{x} f+F(x, p) \cdot \nabla_{p} f=\frac{N+1}{m c^{2}} f(x, p) S \phi, \quad(x, p) \in \Omega \times \mathbb{R}^{N}$,

$$
f(x, p)=g(x, p), \quad(x, p) \in \Sigma^{-} .
$$

The equation (22) can be written

$\alpha f(x, p) e^{-\frac{\phi(x)}{m c^{2}}}+\operatorname{div}_{x}\left(v_{c}(p) f(x, p) e^{-\frac{\phi(x)}{m c^{2}}}\right)+\operatorname{div}_{p}\left(F(x, p) f(x, p) e^{-\frac{\phi(x)}{m c^{2}}}\right)=0$,

and thus we introduce the notion of weak solution for (22), (23) as in Definition 2.1 for any $\phi \in W^{1, \infty}(\Omega), g \in L_{\mathrm{loc}}^{1}\left(\Sigma^{-} ; d \nu^{-}\right)$. We have the classical uniqueness result.

Proposition 2.1. Assume that $\phi$ is smooth (for example $\phi \in W^{2, \infty}(\Omega)$ ), $\alpha>0$ and $g \in L^{\infty}\left(\Sigma^{-} ; d \nu^{-}\right)$. Then there is at most one bounded weak solution for (22), (23).

Proof. Consider $\left(f_{k}\right)_{k \in\{1,2\}}$ two bounded weak solutions for (22), (23) and let $f=f_{1}-f_{2}$. We have

(24) $\alpha f(x, p)+v_{c}(p) \cdot \nabla_{x} f+F(x, p) \cdot \nabla_{p} f=\frac{N+1}{m c^{2}} f(x, p) S \phi, \quad(x, p) \in \Omega \times \mathbb{R}^{N}$,

$$
f(x, p)=0, \quad(x, p) \in \Sigma^{-} .
$$

By (16) we know that $\operatorname{div}_{p} F=-\frac{N}{m c^{2}} S \phi$ and therefore (cf. [4, 17]) we obtain

$$
\begin{aligned}
& 2 \alpha f^{2} e^{-\frac{N+2}{m c^{2}} \phi(x)}+\operatorname{div}_{x}\left(f^{2} e^{-\frac{N+2}{m c^{2}} \phi(x)} v_{c}(p)\right)+\operatorname{div}_{p}\left(f^{2} e^{-\frac{N+2}{m c^{2}} \phi(x)} F(x, p)\right) \\
= & 0, \quad(x, p) \in \Omega \times \mathbb{R}^{N} .
\end{aligned}
$$

After integration on $\Omega \times \mathbb{R}^{N}$ one gets

$$
2 \alpha \int_{\Omega} \int_{\mathbb{R}^{N}} f^{2}(x, p) e^{-\frac{N+2}{m c^{2}} \phi(x)} d p d x+\int_{\Sigma^{+}} f^{2}(x, p) e^{-\frac{N+2}{m c^{2}} \phi(x)} d \nu^{+}=0,
$$

saying that $\left.f\right|_{\Omega \times \mathbb{R}^{N}}=0$ (and also $\left.f\right|_{\Sigma^{+}}=0$ ). 


\subsection{Properties of the characteristics}

We assume that $\phi \in W^{2, \infty}(\Omega)$ is a given function. We start by analyzing the change of variables $(x, p) \rightarrow(X(s ; x, p), P(s ; x, p))$, where $(X, P)$ solves the system of characteristics (18), (19). By using (16) we deduce that the determinant of the jacobian matrix $J(s ; x, p):=\frac{\partial(X(s ; x, p), P(s ; x, p))}{\partial(x, p)}$ satisfies

$$
\frac{d}{d s} \operatorname{det} J(s ; x, p)=-\operatorname{det} J(s ; x, p) \frac{N}{m c^{2}} \frac{d}{d s} \phi(X(s ; x, p)),
$$

and therefore we obtain

$$
\operatorname{det} J(s ; x, p)=e^{-\frac{N}{m c^{2}} \phi(X(s ; x, p))} e^{\frac{N}{m c^{2}} \phi(x)} \neq 0,
$$

saying that

$$
d X(s) d P(s)=e^{-\frac{N}{m c^{2}} \phi(X(s ; x, p))} e^{\frac{N}{m c^{2}} \phi(x)} d x d p .
$$

Consider now the change of variables

$$
\mathcal{O} \ni(s, x, p) \rightarrow(X(s ; x, p), P(s ; x, p)),
$$

where

$$
\mathcal{O}=\cup_{(x, p) \in \Sigma^{-}}(] 0, s_{\text {out }}(x, p)[\times\{x\} \times\{p\}) .
$$

By direct computations we check that

$$
d X d P=\left|v_{c}(p) \cdot n(x)\right| e^{-\frac{N}{m c^{2}} \phi(X(s ; x, p))} e^{\frac{N}{m c^{2}} \phi(x)} d s d \sigma(x) d p .
$$

For any $(x, p) \in \bar{\Omega} \times \mathbb{R}^{N}$ we introduce the energy function

$$
\mathcal{W}_{c}(x, p)=m c^{2}\left(\left(1+\frac{|p|^{2}}{m^{2} c^{2}}\right)^{\frac{1}{2}} e^{\frac{\phi(x)}{m c^{2}}}-1\right) \text {. }
$$

We check easily that $\mathcal{W}_{c}$ is conserved along the characteristics.

Proposition 2.2. Assume that $\phi \in W^{2, \infty}(\Omega)$. Then for any solution of (18) we have

$$
\frac{d}{d s}\left\{\mathcal{W}_{c}(X(s), P(s))\right\}=0, \quad s_{\text {in }}<s<s_{\text {out }}
$$

Observe that

$$
\mathcal{W}_{c}(x, p)=e^{\frac{\phi(x)}{m c^{2}}} \mathcal{E}_{c}(p)+m c^{2}\left(e^{\frac{\phi(x)}{m c^{2}}}-1\right)
$$

where $\mathcal{E}_{c}(p)=m c^{2}\left(\left(1+\frac{|p|^{2}}{m^{2} c^{2}}\right)^{\frac{1}{2}}-1\right)$ is the relativistic kinetic energy. Obviously we have

and

$$
\lim _{c \rightarrow+\infty} \mathcal{E}_{c}(p)=\frac{|p|^{2}}{2 m}, \quad p \in \mathbb{R}^{N}
$$

$$
\lim _{c \rightarrow+\infty} m c^{2}\left(e^{\frac{\phi(x)}{m c^{2}}}-1\right)=\phi(x), \quad x \in \Omega
$$

and therefore the total relativistic energy $\mathcal{W}_{c}$ converges towards the total classical energy $\frac{|p|^{2}}{2 m}+\phi(x)$ when $c$ goes to infinity, as expected. 


\subsection{Properties of the mild solution}

By using the results of the previous paragraph we are ready to establish several properties of the mild solution of (14), (15). We have the following standard results.

Proposition 2.3. Assume that

$$
\phi \in C^{1}(\bar{\Omega}), \nabla_{x} \phi \in W^{1, \infty}(\Omega)^{N}, g \in L_{\mathrm{loc}}^{\infty}\left(\Sigma^{-} ; d \nu^{-}\right) .
$$

Denote by $f$ the mild solution of (14), (15). Then

1) if $g$ is nonnegative, $f$ is nonnegative;

2) $f$ belongs to $L_{\mathrm{loc}}^{\infty}\left(\bar{\Omega} \times \mathbb{R}^{N}\right)$. Moreover, if $g \in L^{\infty}\left(\Sigma^{-} ; d \nu^{-}\right)$, then $f \in$ $L^{\infty}\left(\Omega \times \mathbb{R}^{N}\right)$ and

$$
\|f\|_{L^{\infty}\left(\Omega \times \mathbb{R}^{N}\right)} \leq e^{\frac{N+1}{m c^{2}} \sup _{\Omega} \phi} e^{-\frac{N+1}{m c^{2}} \inf _{\partial \Omega} \phi}\|g\|_{L^{\infty}\left(\Sigma^{-} ; d \nu^{-}\right)} ;
$$

3) for any test function $\psi \in C_{c}^{0}\left(\bar{\Omega} \times \mathbb{R}^{N}\right)$ we have

$$
\begin{aligned}
& \int_{\Omega} \int_{\mathbb{R}^{N}} f(x, p) \psi(x, p) d p d x \\
= & \int_{\Sigma^{-}} g(x, p) e^{-\frac{\phi(x)}{m c^{2}}} \int_{0}^{s_{\text {out }}(x, p)} \psi(X(s ; x, p), P(s ; x, p)) e^{\frac{\phi(X(s ; x, p))}{m c^{2}}} d s d \nu^{-} ;
\end{aligned}
$$

4) $f$ is a weak solution for (14), (15).

Proof. The first statement and the last part of the second one are obvious. Let us check that $f$ is locally bounded. Take $R>0$ and $C>0$ such that

$$
|g(x, p)| \leq C, \text { a.e. }(x, p) \in \Sigma^{-},|p| \leq\left(m^{2} c^{2}+R^{2}\right)^{\frac{1}{2}} e^{\frac{2}{m c^{2}} \sup _{\Omega}|\phi|} .
$$

Consider $(x, p) \in \bar{\Omega} \times \mathbb{R}^{N},|p| \leq R$ such that $s_{\text {in }}(x, p)>-\infty$ (the case $s_{\text {in }}(x, p)=$ $-\infty$ is trivial since $f(x, p)=0)$. By the definition of the mild solution we have

$$
\begin{aligned}
|f(x, p)| & =e^{\frac{N+1}{m c^{2}} \phi(x)} e^{-\frac{N+1}{m c^{2}} \phi\left(X\left(s_{\text {in }} ; x, p\right)\right)}\left|g\left(X\left(s_{\text {in }} ; x, p\right), P\left(s_{\text {in }} ; x, p\right)\right)\right| \\
& \leq e^{\frac{N+1}{m c^{2}} \sup _{\Omega} \phi} e^{-\frac{N+1}{m c^{2}} \inf _{\partial \Omega} \phi}\left|g\left(X\left(s_{\text {in }} ; x, p\right), P\left(s_{\text {in }} ; x, p\right)\right)\right| .
\end{aligned}
$$

By Proposition 2.2 we have also

$$
\left(1+\frac{|p|^{2}}{m^{2} c^{2}}\right)^{\frac{1}{2}} e^{\frac{\phi(x)}{m c^{2}}}=\left(1+\frac{\left|P\left(s_{\text {in }} ; x, p\right)\right|^{2}}{m^{2} c^{2}}\right)^{\frac{1}{2}} e^{\frac{\phi\left(X\left(s_{\text {in }} ; x, p\right)\right)}{m c^{2}}},
$$

and we deduce that

$$
\left|P\left(s_{\text {in }} ; x, p\right)\right| \leq\left(m^{2} c^{2}+R^{2}\right)^{\frac{1}{2}} e^{\frac{2}{m c^{2}} \sup _{\Omega}|\phi|} .
$$

Combining (33), (34), (35) we deduce that

$$
|f(x, p)| \leq e^{\frac{N+1}{m c^{2}} \sup _{\Omega} \phi} e^{-\frac{N+1}{m c^{2}} \inf _{\partial \Omega} \phi} C, \text { a.e. }(x, p) \in \bar{\Omega} \times \mathbb{R}^{N},|p| \leq R,
$$

and thus $f$ is locally bounded. In order to establish the mild formulation (32) observe that $f^{ \pm}:=\max \{0, \pm f\}$ are the mild solutions of $(14),(15)$ corresponding to the boundary conditions $g^{ \pm}:=\max \{0, \pm g\}$ and therefore it is sufficient 
to check (32) when $g \geq 0$ for any nonnegative test function $\psi \in C_{c}^{0}\left(\bar{\Omega} \times \mathbb{R}^{N}\right)$. This follows immediately by using the change of variables (29) and formula (30). Indeed, since $f$ is locally bounded and $\psi$ is compactly supported, $f \psi$ is integrable and we have

$$
\begin{aligned}
& \int_{\Omega} \int_{\mathbb{R}^{N}} f \psi d p d x \\
= & \int_{\Omega} \int_{\mathbb{R}^{N}} f(x, p) \psi(x, p) \mathbf{1}_{\left\{s_{\text {in }}(x, p)>-\infty\right\}} d p d x \\
= & \int_{\Sigma^{-}} \int_{0}^{s_{\text {out }}(x, p)} f(X(s ; x, p), P(s ; x, p)) \psi(X(s ; x, p), P(s ; x, p)) \\
\times & \int_{\Sigma^{-}} g(x, p) e^{-\frac{\phi(x)}{m c^{2}}} \int_{0}^{s_{\text {out }}(x, p)} \psi(X(s ; x, p), P(s ; x, p)) e^{\frac{\phi(X(s ; x, p))}{m c^{2}}} d s d \nu^{-} .
\end{aligned}
$$

For verifying the last statement the idea is to apply the mild formulation (32) with the function

$$
\psi(x, p)=-e^{-\frac{\phi(x)}{m c^{2}}}\left(v_{c}(p) \cdot \nabla_{x} \theta+F(x, p) \cdot \nabla_{p} \theta\right)
$$

for any test function $\theta \in C_{c}^{1}\left(\bar{\Omega} \times \mathbb{R}^{N}\right)$ satisfying $\left.\theta\right|_{\Sigma^{+}}=0$. Observe that for any $(x, p) \in \Sigma^{-}$we have

$$
e^{\frac{\phi(X(s ; x, p))}{m c^{2}}} \psi(X(s ; x, p), P(s ; x, p))=-\frac{d}{d s} \theta(X(s ; x, p), P(s ; x, p)),
$$

and thus

$$
\begin{aligned}
& -\int_{\Omega} \int_{\mathbb{R}^{N}} f(x, p) e^{-\frac{\phi(x)}{m c^{2}}}\left(v_{c}(p) \cdot \nabla_{x} \theta+F(x, p) \cdot \nabla_{p} \theta\right) d p d x \\
= & \int_{\Sigma^{-}} g(x, p) e^{-\frac{\phi(x)}{m c^{2}}} \int_{0}^{s_{\text {out }}(x, p)}\left\{-\frac{d}{d s} \theta(X(s ; x, p), P(s ; x, p))\right\} d s d \nu^{-} .
\end{aligned}
$$

By taking into account that $\left.\theta\right|_{\Sigma^{+}}=0$, we obtain for any $(x, p) \in \Sigma^{-}$such that $s_{\text {out }}(x, p)<+\infty$

$$
\int_{0}^{s_{\text {out }}(x, p)}-\frac{d}{d s}\{\theta(X(s ; x, p), P(s ; x, p))\} d s=\theta(x, p) .
$$

Combining (36), (37) we deduce formally that the weak formulation holds for any test function $\theta \in C_{c}^{1}\left(\bar{\Omega} \times \mathbb{R}^{N}\right)$ such that $\left.\theta\right|_{\Sigma^{+}}=0$. A rigorous proof for checking that the mild solution is a weak solution could be the following. Without loss of generality we assume that $g \geq 0$. For any $\alpha>0$ consider $f_{\alpha}$ the mild solution of the penalized stationary Vlasov problem (22), (23). The solution $f_{\alpha}$ is given by

$$
\begin{aligned}
f_{\alpha}(x, p)= & e^{\frac{N+1}{m c^{2}} \phi(x)} e^{-\frac{N+1}{m c^{2}} \phi\left(X\left(s_{\text {in }} ; x, p\right)\right)} e^{\alpha s_{\text {in }}(x, p)} g\left(X\left(s_{\text {in }} ; x, p\right), P\left(s_{\text {in }} ; x, p\right)\right), \\
& \text { if } s_{\text {in }}(x, p)>-\infty
\end{aligned}
$$


and $f_{\alpha}(x, p)=0$ if $s_{\text {in }}(x, p)=-\infty$. Indeed, the equation (22) can be written (39)

$$
\begin{aligned}
& \alpha f e^{-\frac{N+1}{m c^{2}} \phi(x)}+v_{c}(p) \cdot \nabla_{x}\left(f e^{-\frac{N+1}{m c^{2}} \phi(x)}\right)+F(x, p) \cdot \nabla_{p}\left(f e^{-\frac{N+1}{m c^{2}} \phi(x)}\right)=0, \\
& (x, p) \in \Omega \times \mathbb{R}^{N}
\end{aligned}
$$

and the above formula comes by observing that formally we have

$$
\frac{d}{d s}\left\{e^{\alpha s} f(X(s ; x, p), P(s ; x, p)) e^{-\frac{N+1}{m c^{2}} \phi(X(s ; x, p))}\right\}=0, s_{\text {in }}(x, p)<s<s_{\text {out }}(x, p) \text {. }
$$

As before $f_{\alpha}$ is nonnegative and satisfies the mild formulation

$$
\begin{aligned}
& \int_{\Omega} \int_{\mathbb{R}^{N}} f_{\alpha}(x, p) \psi(x, p) d p d x \\
= & \int_{\Sigma^{-}} g d \nu^{-}(x, p) e^{-\frac{\phi(x)}{m c^{2}}} \int_{0}^{s_{\text {out }}(x, p)} e^{-\alpha s} \psi(X(s ; x, p), P(s ; x, p)) e^{\frac{\phi(X(s ; x, p))}{m c^{2}}} d s
\end{aligned}
$$

for any test function $\psi \in C_{c}^{0}\left(\bar{\Omega} \times \mathbb{R}^{N}\right)$. Now we can verify easily that $f_{\alpha}$ is weak solution for $(22),(23)$. Indeed, for any $\theta \in C_{c}^{1}\left(\bar{\Omega} \times \mathbb{R}^{N}\right)$ satisfying $\left.\theta\right|_{\Sigma^{+}}=0$ consider

$$
\psi(x, p)=e^{-\frac{\phi(x)}{m c^{2}}}\left(\alpha \theta(x, p)-v_{c}(p) \cdot \nabla_{x} \theta-F(x, p) \cdot \nabla_{p} \theta\right),
$$

and observe that

$$
e^{-\alpha s} \psi(X(s ; x, p), P(s ; x, p)) e^{\frac{\phi(X(s ; x, p))}{m c^{2}}}=-\frac{d}{d s}\left\{e^{-\alpha s} \theta(X(s ; x, p), P(s ; x, p))\right\} .
$$

Therefore we obtain

$$
\begin{aligned}
& \int_{\Omega} \int_{\mathbb{R}^{N}} f_{\alpha}(x, p) e^{-\frac{\phi(x)}{m c^{2}}}\left(\alpha \theta(x, p)-v_{c}(p) \cdot \nabla_{x} \theta-F(x, p) \cdot \nabla_{p} \theta\right) d p d x \\
= & \int_{\Sigma^{-}} g d \nu^{-}(x, p) e^{-\frac{\phi(x)}{m c^{2}}} \int_{0}^{s_{\text {out }}}-\frac{d}{d s}\left\{e^{-\alpha s} \theta(X(s ; x, p), P(s ; x, p))\right\} d s,
\end{aligned}
$$

and we are done if we show that

$$
\int_{0}^{s_{\mathrm{out}}(x, p)}-\frac{d}{d s}\left\{e^{-\alpha s} \theta(X(s ; x, p), P(s ; x, p))\right\} d s=\theta(x, p), \quad(x, p) \in \Sigma^{-} .
$$

This is obvious if $s_{\text {out }}(x, p)<+\infty$. In the case $s_{\text {out }}(x, p)=+\infty$ observe that

$$
\begin{aligned}
& \int_{0}^{+\infty}-\frac{d}{d s}\left\{e^{-\alpha s} \theta(X(s ; x, p), P(s ; x, p))\right\} d s \\
= & \theta(x, p)-\lim _{t \rightarrow+\infty}\left\{e^{-\alpha t} \theta(X(t ; x, p), P(t ; x, p))\right\} \\
= & \theta(x, p) .
\end{aligned}
$$

Notice that we have $0 \leq f_{\alpha} \leq f_{\beta} \leq f$ for any $0<\beta \leq \alpha$, where $f$ is the mild solution of (14), (15). Actually we have $f=\sup _{\alpha>0} f_{\alpha}=\lim _{\alpha \searrow 0} f_{\alpha}$. By passing to the limit for $\alpha \searrow 0$ in the weak formulation satisfied by $f_{\alpha}$ one gets easily that $f$ is also a weak solution for (14), (15). 
Remark 2.1. Under the hypotheses of Proposition 2.3 the mild solution $f$ has a locally bounded trace $\gamma^{+} f$ on $\Sigma^{+}$satisfying the Green formula

$$
\begin{aligned}
& -\int_{\Omega} \int_{\mathbb{R}^{N}} f e^{-\frac{\phi(x)}{m c^{2}}}\left(v_{c}(p) \cdot \nabla_{x} \theta+F(x, p) \cdot \nabla_{p} \theta\right) d p d x \\
& +\int_{\Sigma^{+}} \gamma^{+} f e^{-\frac{\phi(x)}{m c^{2}}} \theta(x, p) d \nu^{+}=\int_{\Sigma^{-}} g e^{-\frac{\phi(x)}{m c^{2}}} \theta(x, p) d \nu^{-}
\end{aligned}
$$

for any test function $\theta \in C_{c}^{1}\left(\bar{\Omega} \times \mathbb{R}^{N}\right)$. The trace $\gamma^{+} f$ is given by the same formula as those for $f$ in Definition 2.2, is nonnegative if $g$ is nonnegative, is bounded if $g$ is bounded and we have

$$
\left\|\gamma^{+} f\right\|_{L^{\infty}\left(\Sigma^{+} ; d \nu^{+}\right)} \leq e^{\frac{N+1}{m c^{2}}\left\{\sup _{\partial \Omega} \phi-\inf _{\partial \Omega} \phi\right\}}\|g\|_{L^{\infty}\left(\Sigma^{-} ; d \nu^{-}\right)} .
$$

Analogous results hold for the solutions $\left(f_{\alpha}\right)_{\alpha>0}$.

We intend now to estimate the density $\mu(\cdot)=\int_{\mathbb{R}^{N}} \frac{f(\cdot, p)}{\gamma_{c}(p)} d p$. The crucial point is the conservation of the total energy $\mathcal{W}_{c}$.

Proposition 2.4. Assume that $\phi \in C^{1}(\bar{\Omega}), \nabla_{x} \phi \in W^{1, \infty}(\Omega)^{N}, \phi \geq 0$ on $\partial \Omega$, $g \geq 0$ on $\Sigma^{-}$and that there is a function $H:[0,+\infty[\rightarrow[0,+\infty[$ such that

$$
g(x, p) \leq H\left(\mathcal{W}_{c}(x, p)\right), \quad(x, p) \in \Sigma^{-} .
$$

We denote by $f$ the mild solution of (14), (15). Then we have the inequalities

$$
\begin{gathered}
f(x, p) \leq e^{\frac{N+1}{m c^{2}} \phi(x)} H\left(\mathcal{W}_{c}(x, p)\right) \mathbf{1}_{\left\{\mathcal{W}_{c}(x, p) \geq 0\right\}}, \quad(x, p) \in \Omega \times \mathbb{R}^{N}, \\
\gamma^{+} f(x, p) \leq e^{\frac{N+1}{m c^{2}} \sup _{\partial \Omega} \phi} H\left(\mathcal{W}_{c}(x, p)\right), \quad(x, p) \in \Sigma^{+} .
\end{gathered}
$$

Proof. Since $\phi$ is nonnegative on $\partial \Omega$ we have $\mathcal{W}_{c}(x, p) \geq 0, \forall(x, p) \in \Sigma$. Take $(x, p) \in \Omega \times \mathbb{R}^{N}$ such that $\mathcal{W}_{c}(x, p)<0$. By Proposition 2.2 we have

$$
\left.\mathcal{W}_{c}(X(s ; x, p), P(s ; x, p))=\mathcal{W}_{c}(x, p)<0, \forall s \in\right] s_{\text {in }}(x, p), s_{\text {out }}(x, p)[.
$$

Since $\left.\mathcal{W}_{c}\right|_{\Sigma} \geq 0$ we deduce that $s_{\text {in }}(x, p)=-\infty, f(x, p)=0$ and thus the inequality (43) is trivial. Assume now that $(x, p) \in \Omega \times \mathbb{R}^{N}$ such that $\mathcal{W}_{c}(x, p) \geq$ 0 . As previous we can suppose that $s_{\text {in }}(x, p)>-\infty$ and by the definition of the mild solution and Proposition 2.2 we obtain

$$
\begin{aligned}
f(x, p) & =e^{\frac{N+1}{m c^{2}} \phi(x)} e^{-\frac{N+1}{m c^{2}} \phi\left(X\left(s_{\mathrm{in}} ; x, p\right)\right)} g\left(X\left(s_{\mathrm{in}} ; x, p\right), P\left(s_{\mathrm{in}} ; x, p\right)\right) \\
& \leq e^{\frac{N+1}{m c^{2}} \phi(x)} H\left(\mathcal{W}_{c}\left(X\left(s_{\mathrm{in}} ; x, p\right), P\left(s_{\mathrm{in}} ; x, p\right)\right)\right) \\
& =e^{\frac{N+1}{m c^{2}} \phi(x)} H\left(\mathcal{W}_{c}(x, p)\right) \mathbf{1}_{\left\{\mathcal{W}_{c}(x, p) \geq 0\right\}} .
\end{aligned}
$$

The inequality (44) follows in similar way.

By using Proposition 2.4 we obtain the following estimates for $\mu$ : 
Proposition 2.5. Assume that $\phi \in C^{1}(\bar{\Omega}), \nabla_{x} \phi \in W^{1, \infty}(\Omega)^{N}, \phi \geq 0$ on $\partial \Omega$, $g \geq 0$ on $\Sigma^{-}$and that there is $\delta>N-1, N \geq 2$ such that

$$
g(x, p) \leq \frac{C}{(1+|p|)^{\delta}}, \quad(x, p) \in \Sigma^{-}
$$

for some constant $C>0$. Denote by $f$ the mild solution of $(14),(15), \mu(\cdot)=$ $\int_{\mathbb{R}^{N}} \frac{f(\cdot, p)}{\gamma_{c}(p)} d p, \mu_{R}(\cdot)=\int_{|p| \geq R} \frac{f(\cdot, p)}{\gamma_{c}(p)} d p, \forall R>0$. Then we have

$$
\mu(x) \leq C e^{2 \frac{\phi(x)}{m c^{2}}}, \quad x \in \Omega,
$$

$$
\mu_{R}(x) \leq C \frac{e^{2 \frac{\phi(x)}{m c^{2}}}}{\left(1+\max \left\{0, r_{c}(R) e^{\frac{\phi(x)}{m c^{2}}}+\phi(x)\right\}\right)^{\delta-(N-1)}}
$$

for some constant $C=C\left(m, c, \sup _{\partial \Omega} \phi, N, \delta\right)$ and with

$$
r_{c}(R)=\frac{R^{2}}{m}\left(1+\left(1+\frac{R^{2}}{m^{2} c^{2}}\right)^{\frac{1}{2}}\right)^{-1} .
$$

Proof. We check easily that there is a constant $C_{1}=C_{1}\left(m, c, \sup _{\partial \Omega} \phi\right)$ such that

$$
g(x, p) \leq \frac{C_{1}}{\left(1+\mathcal{W}_{c}(x, p)\right)^{\delta}}, \quad(x, p) \in \Sigma^{-} .
$$

Applying Proposition 2.4 with the function $H(u)=\frac{C_{1}}{(1+u)^{\delta}}, \forall u \geq 0$ yields

$$
f(x, p) \leq e^{\frac{N+1}{m c^{2}} \phi(x)} \frac{C_{1}}{\left(1+\mathcal{W}_{c}(x, p)\right)^{\delta}} \mathbf{1}_{\left\{\mathcal{W}_{c}(x, p) \geq 0\right\}}, \quad(x, p) \in \Omega \times \mathbb{R}^{N} .
$$

The above inequality allows us to estimate $\int_{|p| \geq R} \frac{f(\cdot, p)}{\gamma_{c}(p)} d p$ for any $R \geq 0$. For any fixed $x \in \Omega$ we use the change of variable

$$
m c^{2}\left\{\left(1+\frac{u^{2}}{m^{2} c^{2}}\right)^{\frac{1}{2}} e^{\frac{\phi(x)}{m c^{2}}}-1\right\}=\mathcal{W}
$$

where

$$
\begin{gathered}
u \geq \max \left\{R, m c \sqrt{\left(e^{-2 \frac{\phi(x)}{m c^{2}}}-1\right)}\right\}=: u_{c}^{R}(x), \\
\mathcal{W} \geq \max \left\{0, m c^{2}\left\{\left(1+\frac{R^{2}}{m^{2} c^{2}}\right)^{\frac{1}{2}} e^{\frac{\phi(x)}{m c^{2}}}-1\right\}\right\}=: \mathcal{W}_{c}^{R}(x) .
\end{gathered}
$$

We have the equalities

$$
u=m c\left(\left(\frac{\mathcal{W}}{m c^{2}}+1\right)^{2} e^{-2 \frac{\phi(x)}{m c^{2}}}-1\right)^{\frac{1}{2}}, \quad \mathcal{W} \geq \mathcal{W}_{c}^{R}(x)
$$

and

$$
\frac{d u}{d \mathcal{W}}=\frac{m}{u}\left(\frac{\mathcal{W}}{m c^{2}}+1\right) e^{-2 \frac{\phi(x)}{m c^{2}}}, \quad \mathcal{W} \geq \mathcal{W}_{c}^{R}(x)
$$


Note that we have

$$
\begin{aligned}
& m c^{2}\left(\left(1+\frac{R^{2}}{m^{2} c^{2}}\right)^{\frac{1}{2}} e^{\frac{\phi(x)}{m c^{2}}}-1\right) \\
= & m c^{2}\left(\left(1+\frac{R^{2}}{m^{2} c^{2}}\right)^{\frac{1}{2}}-1\right) e^{\frac{\phi(x)}{m c^{2}}}+m c^{2}\left(e^{\frac{\phi(x)}{m c^{2}}}-1\right) \\
\geq & \frac{R^{2}}{m\left(1+\left(1+\frac{R^{2}}{m^{2} c^{2}}\right)^{\frac{1}{2}}\right.} e^{\frac{\phi(x)}{m c^{2}}}+\phi(x) .
\end{aligned}
$$

We deduce that

$$
\mathcal{W}_{c}^{R}(x) \geq \max \left\{0, r_{c}(R) e^{\frac{\phi(x)}{m c^{2}}}+\phi(x)\right\}
$$

where $r_{c}(R)=\frac{R^{2}}{m\left(1+\left(1+\frac{R^{2}}{m^{2} c^{2}}\right)^{\frac{1}{2}}\right)}, \forall R \geq 0, c>0$. Take now $R \geq 0$ and let us estimate $\int_{|p| \geq R} \frac{f(x, p)}{\gamma_{c}(p)} d p$. We obtain

$$
\begin{aligned}
& \int_{|p| \geq R} \frac{f(x, p)}{\gamma_{c}(p)} d p \\
\leq & C_{1} e^{\frac{N+1}{m c^{2}} \phi(x)} \int_{|p| \geq R} \frac{\mathbf{1}_{\left\{\mathcal{W}_{c}(x, p) \geq 0\right\}}}{\gamma_{c}(p)\left(1+\mathcal{W}_{c}(x, p)\right)^{\delta}} d p \\
\leq & C_{2} e^{\frac{N+1}{m c^{2}} \phi(x)} \int_{u_{c}^{R}(x)}^{+\infty} \frac{u^{N-1} d u}{(1+u)\left(1+m c^{2}\left(\left(1+\frac{u^{2}}{m^{2} c^{2}}\right)^{\frac{1}{2}} e^{\frac{\phi(x)}{m c^{2}}}-1\right)\right)^{\delta}} \\
= & C_{2} e^{\frac{N+1}{m c^{2}} \phi(x)} \int_{\mathcal{W}_{c}^{R}(x)}^{+\infty} \frac{(m c)^{N-2}\left(\left(\frac{\mathcal{W}}{m c^{2}}+1\right)^{2} e^{-2 \frac{\phi(x)}{m c^{2}}}-1\right)^{\frac{N-2}{2}}}{1+m c\left(\left(\frac{\mathcal{W}}{m c^{2}}+1\right)^{2} e^{-2 \frac{\phi(x)}{m c^{2}}}-1\right)^{\frac{1}{2}}} \\
= & C_{2} e^{\frac{N}{m c^{2}} \phi(x)} \int_{\mathcal{W}_{c}^{R}(x)}^{+\infty} \frac{(m c)^{N-2}\left(Q^{2}(\mathcal{W})-1\right)^{\frac{N-2}{2}} m Q(\mathcal{W})}{\left(1+m c\left(Q^{2}(\mathcal{W})-1\right)^{\frac{1}{2}}\right)(1+\mathcal{W})^{\delta}} d \mathcal{W},
\end{aligned}
$$

where we used the notation $Q(\mathcal{W})=\left(\frac{\mathcal{W}}{m c^{2}}+1\right) e^{-\frac{\phi(x)}{m c^{2}}}$. By taking into account that

we deduce that

$$
\sup _{Q \geq 1} \frac{Q}{1+m c\left(Q^{2}-1\right)^{\frac{1}{2}}}<+\infty
$$

$$
\int_{|p| \geq R} \frac{f(x, p)}{\gamma_{c}(p)} d p \leq C_{3} e^{\frac{N}{m c^{2}} \phi(x)} \int_{\mathcal{W}_{c}^{R}(x)}^{+\infty} \frac{\left(Q^{2}(\mathcal{W})-1\right)^{\frac{N-2}{2}}}{(1+\mathcal{W})^{\delta}} d \mathcal{W}
$$




$$
\begin{aligned}
& =C_{3} e^{2 \frac{\phi(x)}{m c^{2}}} \int_{\mathcal{W}_{c}^{R}(x)}^{+\infty} \frac{\left(\left(\frac{\mathcal{W}}{m c^{2}}+1\right)^{2}-e^{2 \frac{\phi(x)}{m c^{2}}}\right)^{\frac{N-2}{2}}}{(1+\mathcal{W})^{\delta}} d \mathcal{W} \\
& \leq C_{3} e^{2 \frac{\phi(x)}{m c^{2}}} \int_{\mathcal{W}_{c}^{R}(x)}^{+\infty}\left(\frac{\mathcal{W}}{m c^{2}}+1\right)^{N-2} \frac{d \mathcal{W}}{(1+\mathcal{W})^{\delta}} \\
& \leq C_{4} e^{2 \frac{\phi(x)}{m c^{2}}} \int_{\mathcal{W}_{c}^{R}(x)}^{+\infty} \frac{d \mathcal{W}}{(1+\mathcal{W})^{\delta-N+2}}
\end{aligned}
$$

In the above computations $C_{2}, C_{3}, C_{4}$ denote some constants depending on $m, c, \sup _{\partial \Omega} \phi, N$. For $R=0$ one gets

$$
\begin{aligned}
\mu(x) & =\int_{\mathbb{R}^{N}} \frac{f(x, p)}{\gamma_{c}(p)} d p \leq C_{4} e^{2 \frac{\phi(x)}{m c^{2}}} \int_{0}^{+\infty} \frac{d \mathcal{W}}{(1+\mathcal{W})^{\delta-N+2}} \\
& \leq C e^{2 \frac{\phi(x)}{m c^{2}}}, \quad x \in \Omega,
\end{aligned}
$$

since we have $\delta>N-1$. Take now $R>0$. We have

$$
\begin{aligned}
\mu_{R}(x) & =\int_{|p| \geq R} \frac{f(x, p)}{\gamma_{c}(p)} d p \leq \frac{C_{4} e^{2 \frac{\phi(x)}{m c^{2}}}}{(\delta-N+1)\left(1+\mathcal{W}_{c}^{R}(x)\right)^{\delta-N+1}} \\
& \leq \frac{C e^{2 \frac{\phi(x)}{m c^{2}}}}{\left(1+\max \left\{0, r_{c}(R) e^{\frac{\phi(x)}{m c^{2}}}+\phi(x)\right\}\right)^{\delta-N+1}} .
\end{aligned}
$$

The above estimates allow us to justify the existence of weak solution for the stationary Nordström-Vlasov system. We intend to investigate the asymptotic behavior of these solutions when the light speed goes to infinity. We need to establish uniform estimates with respect to $c$.

Proposition 2.6. Assume that $\phi \in C^{1}(\bar{\Omega}), \nabla_{x} \phi \in W^{1, \infty}(\Omega)^{N}, \phi \geq 0$ on $\partial \Omega$, $g \geq 0$ on $\Sigma^{-}$and that there is $\delta>2 N, N \geq 2$ such that

$$
g(x, p) \leq \frac{C}{(1+|p|)^{\delta}}, \quad(x, p) \in \Sigma^{-}
$$

for some constant $C>0$. Denote by $f$ the mild solution of (14), (15). Then we have for any $c \geq 1, x \in \Omega$

$$
\begin{gathered}
\int_{\mathbb{R}^{N}} f(x, p) d p \leq C e^{\frac{\phi(x)}{m c^{2}}}\left(1+|\phi(x)|^{\frac{N-2}{2}}\right), \\
\int_{|p| \geq R} f(x, p) d p \leq \frac{C e^{\frac{\phi(x)}{m c^{2}}}\left(1+|\phi(x)|^{\frac{N-2}{2}}\right)}{\left(1+\max \left\{0, r(R) e^{\frac{\phi(x)}{m c^{2}}}+\phi(x)\right\}\right)^{\frac{\delta}{2}-N}}
\end{gathered}
$$


for some constant $C=C\left(m, \sup _{\partial \Omega} \phi, N, \delta\right)$ and with

$$
r(R)=\frac{R^{2}}{m\left(1+\left(1+\frac{R^{2}}{m^{2}}\right)^{\frac{1}{2}}\right)} .
$$

Proof. In the following computations the notation $C$ stands for constants depending on $m,\|\phi\|_{L^{\infty}(\partial \Omega)}, \delta, N$ but not on the light speed. Observe that for any $c>0$ we have the inequalities

$$
\mathcal{E}_{c}(p)=m c^{2}\left(\left(1+\frac{|p|^{2}}{m^{2} c^{2}}\right)^{\frac{1}{2}}-1\right) \leq \frac{|p|^{2}}{2 m}, \quad p \in \mathbb{R}^{N}
$$

and

$$
m c^{2}\left(1-e^{-\frac{\phi(x)}{m c^{2}}}\right) \leq \phi(x), \quad x \in \bar{\Omega} .
$$

Therefore we obtain for any $(x, p) \in \Sigma^{-}$and $c \geq 1$

$$
\begin{aligned}
g(x, p) & \leq \frac{C}{\left(1+\frac{|p|^{2}}{2 m}+\phi(x)\right)^{\frac{\delta}{2}}} \\
& \leq \frac{C}{\left(1+m c^{2}\left(\left(1+\frac{|p|^{2}}{m^{2} c^{2}}\right)^{\frac{1}{2}}-e^{\left.\left.-\frac{\phi(x)}{m c^{2}}\right)\right)^{\frac{\delta}{2}}}\right.\right.} \\
& =\frac{C e^{\frac{\delta \phi(x)}{2 m c^{2}}}}{\left(e^{\frac{\phi(x)}{m c^{2}}}+m c^{2}\left(\left(1+\frac{|p|^{2}}{m^{2} c^{2}}\right)^{\frac{1}{2}} e^{\frac{\phi(x)}{m c^{2}}}-1\right)\right)^{\frac{\delta}{2}}} \\
& \leq \frac{C}{\left(1+\mathcal{W}_{c}(x, p)\right)^{\frac{\delta}{2}}} .
\end{aligned}
$$

By Proposition 2.4 we deduce that

$$
f(x, p) \leq e^{\frac{N+1}{m c^{2}} \phi(x)} \frac{C}{\left(1+\mathcal{W}_{c}(x, p)\right)^{\frac{\delta}{2}}} \mathbf{1}_{\left\{\mathcal{W}_{c}(x, p) \geq 0\right\}}, \quad(x, p) \in \Omega \times \mathbb{R}^{N} .
$$

For any $R \geq 0$ we use one more time the change of variable (48), (49), (50). By using (59) one gets as before

$$
\begin{aligned}
& \int_{|p| \geq R} f(x, p) d p \leq C e^{\frac{N+1}{m c^{2}} \phi(x)} \int_{u_{c}^{R}(x)}^{+\infty} \frac{u^{N-1} d u}{\left(1+m c^{2}\left(\left(1+\frac{u^{2}}{m^{2} c^{2}}\right)^{\frac{1}{2}} e^{\frac{\phi(x)}{m c^{2}}}-1\right)\right)^{\frac{\delta}{2}}} \\
&=C e^{\frac{N+1}{m c^{2}} \phi(x)} \int_{\mathcal{W}_{c}^{R}(x)}^{+\infty} \frac{(m c)^{N-2}\left(\left(\frac{\mathcal{W}}{m c^{2}}+1\right)^{2} e^{-2 \frac{\phi(x)}{m c^{2}}}-1\right)^{\frac{N-2}{2}}}{(1+\mathcal{W})^{\frac{\delta}{2}}} \\
& \times m\left(\frac{\mathcal{W}}{m c^{2}}+1\right) e^{-2 \frac{\phi(x)}{m c^{2}}} d \mathcal{W}, \quad x \in \Omega .
\end{aligned}
$$


Observe that for any $\mathcal{W} \geq \mathcal{W}_{c}^{R}(x)$ and $c \geq 1$ we have

$m c\left(\left(\frac{\mathcal{W}}{m c^{2}}+1\right)^{2} e^{-2 \frac{\phi(x)}{m c^{2}}}-1\right)^{\frac{1}{2}}=m c e^{-\frac{\phi(x)}{m c^{2}}}\left(\frac{\mathcal{W}}{m c^{2}}\left(\frac{\mathcal{W}}{m c^{2}}+2\right)+1-e^{2 \frac{\phi(x)}{m c^{2}}}\right)^{\frac{1}{2}}$

$$
\begin{aligned}
& \leq m c e^{-\frac{\phi(x)}{m c^{2}}}\left(\frac{\mathcal{W}}{m c^{2}}\left(\frac{\mathcal{W}}{m c^{2}}+2\right)-\frac{2 \phi(x)}{m c^{2}}\right)^{\frac{1}{2}} \\
& \leq C e^{-\frac{\phi(x)}{m c^{2}}}\left(\mathcal{W}+1+|\phi(x)|^{\frac{1}{2}}\right) .
\end{aligned}
$$

Combining (60), (61) yields

$$
\int_{|p| \geq R} f(x, p) d p \leq C e^{\frac{\phi(x)}{m c^{2}}} \int_{\mathcal{W}_{c}^{R}(x)}^{+\infty} \frac{(1+\mathcal{W})^{N-2}+|\phi(x)|^{\frac{N-2}{2}}}{(1+\mathcal{W})^{\frac{\delta}{2}-1}} d \mathcal{W} .
$$

For $R=0$ one gets

$$
\int_{\mathbb{R}^{N}} f(x, p) d p \leq C e^{\frac{\phi(x)}{m c^{2}}}\left(1+|\phi(x)|^{\frac{N-2}{2}}\right), x \in \Omega, c \geq 1
$$

since we have $\delta>2 N$. By taking into account that for any $R>0, x \in \Omega, c \geq 1$ we have

we obtain

$$
\mathcal{W}_{c}^{R}(x) \geq \max \left\{0, r(R) e^{\frac{\phi(x)}{m c^{2}}}+\phi(x)\right\}
$$

$$
\int_{|p| \geq R} f(x, p) d p \leq \frac{C e^{\frac{\phi(x)}{m c^{2}}}\left(1+|\phi(x)|^{\frac{N-2}{2}}\right)}{\left(1+\max \left\{0, r(R) e^{\frac{\phi(x)}{m c^{2}}}+\phi(x)\right\}\right)^{\frac{\delta}{2}-N}} .
$$

Remark 2.2. For any $\alpha>0$ denote by $f_{\alpha}$ the mild solution of $(22),(23)$ and by $f$ the mild solution of (14), (15). Since $0 \leq f_{\alpha} \leq f$ we deduce that the conclusions of Propositions 2.5, 2.6 hold true for $f_{\alpha}$, uniformly with respect to $\alpha>0$.

\section{Fixed point application}

We intend to show the existence of weak solution for the Nordström-Vlasov equations by using the Schauder fixed point theorem. We assume that $\varphi_{0}$ is a nonnegative smooth function on the boundary $\partial \Omega$ and we consider $\phi_{0}$ the solution of the problem

$$
-\Delta_{x} \phi_{0}=0, \quad x \in \Omega, \quad \phi_{0}(x)=\varphi_{0}(x), \quad x \in \partial \Omega .
$$

If $\Omega$ is of class $C^{3}$ and $\varphi_{0}$ belongs to $W^{3-\frac{1}{q}, q}(\partial \Omega)$ for some $q>N$, then $\nabla_{x} \phi_{0} \in W^{2, q}(\Omega) \subset W^{1, \infty}(\Omega)$. In order to use the mild formulation we need to regularize the field $\nabla_{x} \phi$. Since we want to preserve some information about the trace of $\phi$ on the boundary $\partial \Omega$ it is convenient to use some special regularization procedure. Let us introduce some notations. For any $\varepsilon>0$ consider

$$
\mathcal{O}_{\varepsilon}=\{x \in \bar{\Omega}: \operatorname{dist}(x, \partial \Omega)<\varepsilon\} .
$$


Since $\Omega$ is bounded and smooth, there is $\varepsilon_{\Omega}>0$ and a smooth function $D: \bar{\Omega} \rightarrow$ $\mathbb{R}$ such that $D(x)=\operatorname{dist}(x, \partial \Omega), \forall x \in \mathcal{O}_{\varepsilon_{\Omega}}, \nu=-\nabla_{x} D$ is regular and bounded in $\bar{\Omega}$ and $\nu(x)=n\left(P_{\partial \Omega}(x)\right)$ for any $x \in \mathcal{O}_{\varepsilon_{\Omega}}$, where $P_{\partial \Omega}$ is the projection on $\partial \Omega$ and $n$ is the unit outward normal on $\partial \Omega$. In particular $\nu(x)=n(x)$ for any $x \in \partial \Omega$. Consider $\zeta \in C_{c}^{\infty}\left(\mathbb{R}^{N}\right), \zeta \geq 0, \operatorname{supp} \zeta \subset\left\{x \in \mathbb{R}^{N}:|x| \leq 1\right\}$, $\int_{\mathbb{R}^{N}} \zeta(x) d x=1, \zeta_{\varepsilon}(\cdot)=\frac{1}{\varepsilon^{N}} \zeta(\dot{\bar{\varepsilon}})$ for any $\varepsilon>0$. Following the construction in $[27]$ for any $\phi \in H_{0}^{1}(\Omega)$ we define

$$
\begin{aligned}
R_{\varepsilon} \phi(x) & =\int_{\mathbb{R}^{N}} \zeta_{\varepsilon}(y) \bar{\phi}(x+2 \varepsilon \nu(x)-y) d y \\
& =\int_{\mathbb{R}^{N}} \zeta_{\varepsilon}(x+2 \varepsilon \nu(x)-y) \bar{\phi}(y) d y, \quad x \in \bar{\Omega},
\end{aligned}
$$

where $\bar{\phi}(x)=\phi(x), x \in \Omega$ and $\bar{\phi}(x)=0, x \in \mathbb{R}^{N}-\Omega$. If $\Omega$ is of class $C^{3}$, then $\nu$ is of class $C^{2}$ and therefore $R_{\varepsilon} \phi \in C^{2}\left(\mathbb{R}^{N}\right)$. Observe that for $\varepsilon$ small enough we have $R_{\varepsilon} \phi(x)=0, \forall x \in \mathcal{O}_{\varepsilon}$ and thus $R_{\varepsilon} \phi \in C_{c}^{2}(\Omega)$. Moreover we have $\lim _{\varepsilon \backslash 0} R_{\varepsilon} \phi=\phi$ in $H^{1}(\Omega)$. For any $c>0$ we define the fixed point map $\mathcal{F}_{c, \varepsilon}$ as follows: for $\phi \in H_{0}^{1}(\Omega)$ consider $\mathcal{F}_{c, \varepsilon} \phi=\tilde{\phi}$ where

- $f$ is the mild solution for the stationary regularized Vlasov problem

$$
\begin{gathered}
\quad \varepsilon f(x, p)+v_{c}(p) \cdot \nabla_{x} f-\left(v_{c}(p) \cdot \nabla_{x}\left(R_{\varepsilon} \phi+\phi_{0}\right) \frac{p}{m c^{2}}+\frac{\nabla_{x}\left(R_{\varepsilon} \phi+\phi_{0}\right)}{\gamma_{c}(p)}\right) \cdot \nabla_{p} f \\
=\frac{N+1}{m c^{2}} f(x, p) v_{c}(p) \cdot \nabla_{x}\left(R_{\varepsilon} \phi+\phi_{0}\right), \quad(x, p) \in \Omega \times \mathbb{R}^{N}, \\
\quad f(x, p)=g(x, p), \quad(x, p) \in \Sigma^{-} ;
\end{gathered}
$$

- $\tilde{\phi}$ is the solution of the Poisson problem

$$
\begin{gathered}
-\Delta_{x} \tilde{\phi}=-\mu(x), \quad x \in \Omega, \\
\tilde{\phi}(x)=0, \quad x \in \partial \Omega,
\end{gathered}
$$

with $\mu(\cdot)=\int_{\mathbb{R}^{N}} \frac{f(\cdot, p)}{\gamma_{c}(p)} d p$.

The properties of the map $\mathcal{F}_{c, \varepsilon}$ are summed up in the following straightforward result:

Proposition 3.1. Assume that $\Omega$ is regular, $\varphi_{0} \in W^{3-\frac{1}{q}, q}(\partial \Omega)$ for some $q>$ $N, \varphi_{0} \geq 0$ on $\partial \Omega, g \geq 0$ on $\Sigma^{-}$such that

$$
g(x, p) \leq \frac{C}{(1+|p|)^{\delta}}, \quad(x, p) \in \Sigma^{-}
$$

for some constants $C>0, \delta>N-1$ with $N \geq 2$. Then

1) there is a constant $C_{c}=C\left(m, c, \sup _{\partial \Omega} \varphi_{0}, N, \delta\right)$ such that

$$
\left\|\mathcal{F}_{c, \varepsilon} \phi\right\|_{H^{1}(\Omega)} \leq C_{c}, \forall \phi \in H_{0}^{1}(\Omega), \phi \leq 0 ;
$$

2) the map $\mathcal{F}_{c, \varepsilon}$ is continuous with respect to the weak topology of $H^{1}(\Omega)$;

3) there is a fixed point for the application $\mathcal{F}_{c, \varepsilon}$. 
Proof. 1) For any $\phi \in H_{0}^{1}(\Omega), \phi \leq 0$ we have $R_{\varepsilon} \phi \leq 0$. By Proposition 2.5 and Remark 2.2 we know that

$$
0 \leq \mu(x) \leq C_{1} e^{2 \frac{\phi_{0}(x)+R_{\varepsilon} \phi(x)}{m c^{2}}} \leq C_{1} e^{\frac{2}{m c^{2}} \sup _{\partial \Omega} \varphi_{0}}=: C_{2}, \quad x \in \Omega
$$

for some constant $C_{1}=C_{1}\left(m, c, \sup _{\partial \Omega} \varphi_{0}, N, \delta\right)$. In particular we deduce that $\|\mu\|_{L^{2}(\Omega)} \leq C_{2}(\operatorname{meas}(\Omega))^{1 / 2}$ which implies that

$$
\left\|\mathcal{F}_{c, \varepsilon} \phi\right\|_{H^{1}(\Omega)}=\|\tilde{\phi}\|_{H^{1}(\Omega)} \leq C_{3}\left(m, c, \sup _{\partial \Omega} \varphi_{0}, N, \delta\right)=: C_{c} .
$$

2) The arguments are standard. Take $\left(\phi_{k}\right)_{k} \subset H_{0}^{1}(\Omega)$ such that $\lim _{k \rightarrow+\infty} \phi_{k}$ $=\phi$ weakly in $H^{1}(\Omega)$. By weak convergence we have

$$
R_{\varepsilon} \phi_{k}(x) \rightarrow R_{\varepsilon} \phi(x), \quad \nabla_{x} R_{\varepsilon} \phi_{k}(x) \rightarrow \nabla_{x} R_{\varepsilon} \phi(x), \quad x \in \Omega .
$$

We check easily that

$$
\sup _{k}\left\{\left\|R_{\varepsilon} \phi_{k}\right\|_{L^{\infty}(\Omega)}+\left\|\nabla_{x} R_{\varepsilon} \phi_{k}\right\|_{L^{\infty}(\Omega)}\right\}<+\infty
$$

and by using the dominated convergence theorem we have

$$
\lim _{k \rightarrow+\infty} \nabla_{x} R_{\varepsilon} \phi_{k}=\nabla_{x} R_{\varepsilon} \phi \text { strongly in } L^{2}(\Omega)^{N} .
$$

Denote by $\left(f_{k}\right)_{k}, f$ the mild solutions of $(67),(68)$ corresponding to the fields $\left(\nabla_{x} R_{\varepsilon} \phi_{k}\right)_{k}$, respectively $\nabla_{x} R_{\varepsilon} \phi$. By Proposition 2.3 we know that $\left(f_{k}\right)_{k}$ are also weak solutions and therefore, for any test function $\theta \in C_{c}^{1}\left(\bar{\Omega} \times \mathbb{R}^{N}\right)$, $\left.\theta\right|_{\Sigma^{+}}=0$ we can write

$$
\begin{aligned}
& \int_{\Omega} \int_{\mathbb{R}^{N}} f_{k}(x, p) e^{-\frac{R_{\varepsilon} \phi_{k}(x)+\phi_{0}(x)}{m c^{2}}}\left(\varepsilon \theta(x, p)-v_{c}(p) \cdot \nabla_{x} \theta-F_{k}(x, p) \cdot \nabla_{p} \theta\right) d p d x \\
= & \int_{\Sigma^{-}} g(x, p) e^{-\frac{\varphi_{0}(x)}{m c^{2}}} \theta(x, p) d \nu^{-}
\end{aligned}
$$

where $F_{k}(x, p)=-\left(v_{c}(p) \cdot \nabla_{x}\left(R_{\varepsilon} \phi_{k}+\phi_{0}\right) \frac{p}{m c^{2}}+\frac{\nabla_{x}\left(R_{\varepsilon} \phi_{k}+\phi_{0}\right)}{\gamma_{c}(p)}\right)$. By Proposition 2.3 we have

$$
\sup _{k}\left\|f_{k}\right\|_{L^{\infty}\left(\Omega \times \mathbb{R}^{N}\right)} \leq \sup _{k} e^{\frac{N+1}{m c^{2}} \sup _{\Omega}\left(R_{\varepsilon} \phi_{k}+\phi_{0}\right)}\|g\|_{L^{\infty}\left(\Sigma^{-} ; d \nu^{-}\right)}<+\infty,
$$

and therefore we can extract a sequence $\left(k_{i}\right)_{i}$ such that $f_{k_{i}} \rightarrow \tilde{f}$ weakly $\star$ in $L^{\infty}\left(\Omega \times \mathbb{R}^{N}\right)$. By using (73), (74), (75) we can pass easily to the limit with respect to $i$ in the weak formulations (76) written for $k=k_{i}$ and we deduce that $\tilde{f}$ is a weak solution for (67), (68). By Proposition 2.1 we deduce that $\tilde{f}=f$. Actually all the sequence $\left(f_{k}\right)_{k}$ converges weakly $\star$ in $L^{\infty}\left(\Omega \times \mathbb{R}^{N}\right)$ towards $f$. Denote by $\left(\mu_{k}\right)_{k}$ and $\mu$ the densities given by

$$
\mu_{k}(\cdot)=\int_{\mathbb{R}^{N}} \frac{f_{k}(\cdot, p)}{\gamma_{c}(p)} d p, \forall k, \mu(\cdot)=\int_{\mathbb{R}^{N}} \frac{f(\cdot, p)}{\gamma_{c}(p)} d p .
$$


By using Proposition 2.5 and by taking into account that $\sup _{k}\left\|R_{\varepsilon} \phi_{k}+\phi_{0}\right\|_{L^{\infty}(\Omega)}$ $<+\infty$ we deduce that

$$
\sup _{k \geq 1}\left\|\mu_{k}\right\|_{L^{\infty}(\Omega)}<+\infty, \lim _{R \rightarrow+\infty} \sup _{k \geq 1}\left\|\int_{|p| \geq R} \frac{f_{k}(\cdot, p)}{\gamma_{c}(p)} d p\right\|_{L^{\infty}(\Omega)}=0
$$

and we obtain easily that $\mu_{k} \rightarrow \mu$ weakly in $L^{s}(\Omega), \forall 1 \leq s<+\infty$ and $\mu_{k} \rightarrow \mu$ weakly $\star$ in $L^{\infty}(\Omega)$. Consider $\left(\tilde{\phi}_{k}\right)_{k}, \tilde{\phi}$ the solutions of the problems

$$
\begin{gathered}
-\Delta_{x} \tilde{\phi}_{k}=-\mu_{k}(x), \quad x \in \Omega, \quad \tilde{\phi}_{k}(x)=0, \quad x \in \partial \Omega, \\
-\Delta_{x} \tilde{\phi}=-\mu(x), \quad x \in \Omega, \quad \tilde{\phi}(x)=0, \quad x \in \partial \Omega .
\end{gathered}
$$

Since $\left(\mu_{k}\right)_{k}$ is bounded in $L^{2}(\Omega),\left(\tilde{\phi}_{k}\right)_{k}$ is bounded in $H^{2}(\Omega)$ and thus we can extract a subsequence $\left(\tilde{\phi}_{k_{i}}\right)_{i}$ which converges in $H^{1}(\Omega)$. By using the convergence $\mu_{k_{i}} \rightarrow \mu$ weakly $\star$ in $L^{\infty}(\Omega)$ we deduce that $\lim _{i \rightarrow+\infty} \tilde{\phi}_{k_{i}}=\tilde{\phi}$ in $H^{1}(\Omega)$. In fact all the sequence $\left(\tilde{\phi}_{k}\right)_{k}=\left(\mathcal{F}_{c, \varepsilon} \phi_{k}\right)_{k}$ converges strongly in $H^{1}(\Omega)$ towards $\tilde{\phi}=\mathcal{F}_{c, \varepsilon} \phi$. In particular $\mathcal{F}_{c, \varepsilon}$ is continuous with respect to the weak topology of $H^{1}(\Omega)$.

3) We consider the set $\mathcal{X}_{c, \varepsilon}=\left\{\phi \in H_{0}^{1}(\Omega):\|\phi\|_{H^{1}(\Omega)} \leq C_{c}, \phi \leq 0\right\}$ which is convex and compact with respect to the weak topology of $H^{1}(\Omega)$. Observe also that $\mathcal{F}_{c, \varepsilon}\left(\mathcal{X}_{c, \varepsilon}\right) \subset \mathcal{X}_{c, \varepsilon}$. Indeed, by construction $\tilde{\phi}=\mathcal{F}_{c, \varepsilon} \phi \in H_{0}^{1}(\Omega)$ and by the first point we have $\|\tilde{\phi}\|_{H^{1}(\Omega)} \leq C_{c}$. Since $-\Delta_{x} \tilde{\phi}=-\mu(x) \leq 0, x \in \Omega$ and $\left.\tilde{\phi}\right|_{\partial \Omega}=0$ we have $\sup _{\Omega} \tilde{\phi} \leq 0$. We conclude by the Schauder fixed point theorem.

\section{The stationary Nordström-Vlasov equations}

By passing to the limit with respect to $\varepsilon \searrow 0$ we obtain the existence of weak solution for the stationary Nordström-Vlasov system as stated in Theorem 1.1.

Proof of Theorem 1.1. For any fixed $c>0$ take $\left(\varepsilon_{k}\right)_{k \geq 1}$ a decreasing sequence converging towards 0 and consider $\varphi_{0, k} \in W^{3-\frac{1}{q}, q}(\partial \Omega)$ for some $q>N$ such that

$\lim _{k \rightarrow+\infty} \varphi_{0, k}=\varphi_{0}$ in $H^{1 / 2}(\partial \Omega), \sup _{k \geq 1}\left\|\varphi_{0, k}\right\|_{L^{\infty}(\partial \Omega)} \leq\left\|\varphi_{0}\right\|_{L^{\infty}(\partial \Omega)}, \varphi_{0, k} \geq 0, \forall k$.

By Proposition 3.1 there is $\left(f_{c, k}, \phi_{c, k}\right)$ solution for

$$
\begin{aligned}
& \quad \varepsilon_{k} f_{c, k}+v_{c}(p) \cdot \nabla_{x} f_{c, k}-\left(v_{c}(p) \cdot \nabla_{x}\left(R_{\varepsilon_{k}} \phi_{c, k}+\phi_{0, k}\right) \frac{p}{m c^{2}}+\frac{\nabla_{x}\left(R_{\varepsilon_{k}} \phi_{c, k}+\phi_{0, k}\right)}{\gamma_{c}(p)}\right) \cdot \nabla_{p} f_{c, k} \\
& =\frac{N+1}{m c^{2}} f_{c, k}(x, p) v_{c}(p) \cdot \nabla_{x}\left(R_{\varepsilon_{k}} \phi_{c, k}+\phi_{0, k}\right), \quad(x, p) \in \Omega \times \mathbb{R}^{N}, \\
& \text { (81) } \quad-\Delta_{x} \phi_{c, k}=-\mu_{c, k}(x)=-\int_{\mathbb{R}^{N}} \frac{f_{c, k}(x, p)}{\gamma_{c}(p)} d p,-\Delta_{x} \phi_{0, k}=0, \quad x \in \Omega,
\end{aligned}
$$


(82)

$$
f_{c, k}(x, p)=g(x, p), \quad(x, p) \in \Sigma^{-}, \quad \phi_{c, k}(x)=0, \quad \phi_{0, k}(x)=\varphi_{0, k}(x), \quad x \in \partial \Omega
$$

such that

$$
\begin{gathered}
0 \leq \phi_{0, k}(x) \leq\left\|\varphi_{0, k}\right\|_{L^{\infty}(\partial \Omega)} \leq\left\|\varphi_{0}\right\|_{L^{\infty}(\partial \Omega)}, \quad \phi_{c, k}(x) \leq 0, \forall x \in \Omega, \forall k \geq 1, \\
0 \leq f_{c, k}(x, p) \leq e^{\frac{N+1}{m c^{2}}\left\|\varphi_{0}\right\|_{L^{\infty}(\partial \Omega)}\|g\|_{L^{\infty}\left(\Sigma^{-}\right)},(x, p) \in \Sigma^{-}, k \geq 1,} \\
\sup _{k \geq 1}\left\|\int_{\mathbb{R}^{N}} \frac{f_{c, k}(\cdot, p)}{\gamma_{c}(p)} d p\right\|_{L^{\infty}(\Omega)} \leq C(c) e^{\frac{2}{m c^{2}}\left\|\varphi_{0}\right\|_{L^{\infty}(\partial \Omega)}}
\end{gathered}
$$

for some constant depending on $c$. In particular we have

$$
\sup _{k \geq 1}\left\|\mu_{c, k}\right\|_{L^{N+1}(\Omega)}<+\infty,
$$

and thus

$$
\sup _{k \geq 1}\left\|\phi_{c, k}\right\|_{L^{\infty}(\Omega)} \leq C \sup _{k \geq 1}\left\|\phi_{c, k}\right\|_{W^{1, N+1}(\Omega)} \leq C \sup _{k \geq 1}\left\|\mu_{c, k}\right\|_{L^{N+1}(\Omega)}<+\infty .
$$

By using Proposition 2.5 we deduce easily that

$$
\lim _{R \rightarrow+\infty} \sup _{k \geq 1}\left\|\int_{|p| \geq R} \frac{f_{c, k}(\cdot, p)}{\gamma_{c}(p)} d p\right\|_{L^{\infty}(\Omega)}=0 .
$$

We can assume (after extraction eventually) that

$$
\begin{gathered}
\lim _{k \rightarrow+\infty} f_{c, k}=f_{c}, \text { weakly } \star \text { in } L^{\infty}\left(\Omega \times \mathbb{R}^{N}\right), \\
\lim _{k \rightarrow+\infty} \mu_{c, k}=\mu_{c}:=\int_{\mathbb{R}^{N}} \frac{f_{c}(\cdot, p)}{\gamma_{c}(p)} d p, \text { weakly } \star \text { in } L^{\infty}(\Omega), \\
\lim _{k \rightarrow+\infty} \phi_{c, k}=\phi_{c}, \text { strongly in } H^{1}(\Omega), \\
\lim _{k \rightarrow+\infty} \phi_{0, k}=\phi_{0}, \text { strongly in } H^{1}(\Omega),
\end{gathered}
$$

where $\phi_{0}$ is the solution of (65). We check easily that $\left(f_{c}, \phi_{c}+\phi_{0}\right)$ is a weak solution of the stationary Nordström-Vlasov system. Observe also that

$$
0 \leq f_{c} \leq e^{\frac{N+1}{m c^{2}}\left\|\varphi_{0}\right\|_{L^{\infty}(\partial \Omega)}}\|g\|_{L^{\infty}\left(\Sigma^{-}\right)}, \lim _{R \rightarrow+\infty}\left\|\int_{|p| \geq R} \frac{f_{c}(\cdot, p)}{\gamma_{c}(p)} d p\right\|_{L^{\infty}(\Omega)}=0 .
$$

Assume now that $\delta>2 N$ with $N \in\{2,3\}$. By using Proposition 2.6 we have for any $c \geq 1$

$$
\begin{aligned}
\int_{\mathbb{R}^{N}} f_{c, k}(x, p) d p & \leq C_{1} e^{\frac{\left\|\varphi_{0}\right\|_{L} \infty(\partial \Omega)}{m c^{2}}}\left(1+\left|R_{\varepsilon_{k}} \phi_{c, k}(x)+\phi_{0, k}(x)\right|^{\frac{N-2}{2}}\right) \\
& \leq C_{2}\left(1+\left|R_{\varepsilon_{k}} \phi_{c, k}(x)\right|^{\frac{N-2}{2}}\right), \quad x \in \Omega, \quad k \geq 1
\end{aligned}
$$


for some constants $C_{1}, C_{2}$ depending on $m,\left\|\varphi_{0}\right\|_{L^{\infty}(\partial \Omega)}, N, \delta$ but not on $c$. If $N=2$ the inequality (83) gives the uniform estimates

$$
\begin{aligned}
\sup _{k \geq 1, c \geq 1}\left\|\mu_{c, k}\right\|_{L^{\infty}(\Omega)} \leq & \sup _{k \geq 1, c \geq 1}\left\|\int_{\mathbb{R}^{2}} f_{c, k}(\cdot, p) d p\right\|_{L^{\infty}(\Omega)} \leq 2 C_{2}, \\
\sup _{k \geq 1, c \geq 1}\left\|\phi_{c, k}\right\|_{L^{\infty}(\Omega)} & \leq C(\Omega) \sup _{k \geq 1, c \geq 1}\left\|\phi_{c, k}\right\|_{W^{1,3}(\Omega)} \\
& \leq C(\Omega) \sup _{k \geq 1, c \geq 1}\left\|\mu_{c, k}\right\|_{L^{3}(\Omega)}<+\infty .
\end{aligned}
$$

Combining (85), (58) yields

$$
\lim _{R \rightarrow+\infty} \sup _{k \geq 1, c \geq 1}\left\|\int_{|p| \geq R} f_{c, k}(\cdot, p) d p\right\|_{L^{\infty}(\Omega)}=0 .
$$

By passing to the limit with respect to $k$ we obtain that $f_{c}=\mathrm{w} \star \lim _{k \rightarrow+\infty} f_{c, k}$ satisfies

$$
\sup _{c \geq 1}\left\|\int_{\mathbb{R}^{2}} f_{c}(\cdot, p) d p\right\|_{L^{\infty}(\Omega)}<+\infty, \lim _{R \rightarrow+\infty} \sup _{c \geq 1}\left\|\int_{|p| \geq R} f_{c}(\cdot, p) d p\right\|_{L^{\infty}(\Omega)}=0 .
$$

We analyze now the case $N=3$. We have from (83)

$$
\int_{\mathbb{R}^{3}} f_{c, k}(x, p) d p \leq C_{2}\left(1+\left|R_{\varepsilon_{k}} \phi_{c, k}(x)\right|^{1 / 2}\right), \quad x \in \Omega, k \geq 1, c \geq 1 .
$$

We deduce that for any $s>3, c \geq 1$ we have

$$
\begin{aligned}
\left\|\mu_{c, k}\right\|_{L^{s}(\Omega)}^{s} & \leq C_{3}\left(1+\left\|R_{\varepsilon_{k}} \phi_{c, k}\right\|_{L^{s / 2}(\Omega)}^{s / 2}\right) \\
& \leq C_{4}\left(1+\left\|\phi_{c, k}\right\|_{L^{s / 2}(\Omega)}^{s / 2}\right) \\
& \leq C_{5}\left(1+\left\|\phi_{c, k}\right\|_{L^{s}(\Omega)}^{s / 2}\right) \\
& \leq C_{6}\left(1+\left\|\mu_{c, k}\right\|_{L^{s}(\Omega)}^{s / 2}\right)
\end{aligned}
$$

for some constants $C_{3}, C_{4}, C_{5}, C_{6}$ not depending on $k \geq 1$ and $c \geq 1$ and therefore we obtain $\sup _{k \geq 1, c \geq 1}\left\|\mu_{c, k}\right\|_{L^{s}(\Omega)}<+\infty$. Finally one gets

$$
\begin{aligned}
\sup _{k \geq 1, c \geq 1}\left\|\phi_{c, k}\right\|_{L^{\infty}(\Omega)} & \leq C_{7}(\Omega) \sup _{k \geq 1, c \geq 1}\left\|\phi_{c, k}\right\|_{W^{1, s}(\Omega)} \\
& \leq C_{8} \sup _{k \geq 1, c \geq 1}\left\|\mu_{c, k}\right\|_{L^{s}(\Omega)}<+\infty .
\end{aligned}
$$

From (87), (58) we deduce that

$$
\begin{gathered}
\sup _{k \geq 1, c \geq 1}\left\|\int_{\mathbb{R}^{3}} f_{c, k}(\cdot, p) d p\right\|_{L^{\infty}(\Omega)}<+\infty, \\
\lim _{R \rightarrow+\infty} \sup _{k \geq 1, c \geq 1}\left\|\int_{|p| \geq R} f_{c, k}(\cdot, p) d p\right\|_{L^{\infty}(\Omega)}=0 .
\end{gathered}
$$


By passing to the limit with respect to $k$ we obtain that $f_{c}=\mathrm{w} \star \lim _{k \rightarrow+\infty} f_{c, k}$ satisfies

$$
\sup _{c \geq 1}\left\|\int_{\mathbb{R}^{3}} f_{c}(\cdot, p) d p\right\|_{L^{\infty}(\Omega)}<+\infty, \lim _{R \rightarrow+\infty} \sup _{c \geq 1}\left\|\int_{|p| \geq R} f_{c}(\cdot, p) d p\right\|_{L^{\infty}(\Omega)}=0 .
$$

For any $c>0$ we proved the existence of weak solution for the stationary Nordström-Vlasov system. A natural question is what happens if the light speed $c$ goes to infinity. We can prove the convergence towards a weak solution of the Vlasov-Poisson system for stellar dynamics as stated in Theorem 1.2.

Proof of Theorem 1.2. Take $\left(c_{k}\right)_{k}$ an increasing sequence such that $\lim _{k \rightarrow+\infty} c_{k}$ $=+\infty$. For any $k$ we consider the solution $\left(f_{k}, \phi_{k}\right):=\left(f_{c_{k}}, \phi_{c_{k}}\right)$ constructed in Theorem 1.1.

$$
\begin{gathered}
v_{k}(p) \cdot \nabla_{x} f_{k}-\left(v_{k}(p) \cdot \nabla_{x} \phi_{k} \frac{p}{m c_{k}^{2}}+\frac{\nabla_{x} \phi_{k}}{\gamma_{k}(p)}\right) \cdot \nabla_{p} f_{k} \\
=\frac{N+1}{m c_{k}^{2}} f_{k}(x, p) v_{k}(p) \cdot \nabla_{x} \phi_{k}, \quad(x, p) \in \Omega \times \mathbb{R}^{N}, \\
-\Delta_{x} \phi_{k}=-\mu_{k}(x):=-\int_{\mathbb{R}^{N}} \frac{f_{k}(x, p)}{\gamma_{k}(p)} d p, \quad x \in \Omega, \\
f_{k}(x, p)=g(x, p), \quad(x, p) \in \Sigma^{-}, \phi_{k}(x)=\varphi_{0}(x), \quad x \in \partial \Omega,
\end{gathered}
$$

where $\gamma_{k}(p)=\gamma_{c_{k}}(p), v_{k}(p)=v_{c_{k}}(p), p \in \mathbb{R}^{N}$. By Theorem 1.1 we also know that

$$
\begin{gathered}
\sup _{k \geq 1}\left\|f_{k}\right\|_{L^{\infty}\left(\Omega \times \mathbb{R}^{N}\right)} \leq e^{\frac{N+1}{m c^{2}}\left\|\varphi_{0}\right\|_{L^{\infty}(\partial \Omega)}\|g\|_{L^{\infty}\left(\Sigma^{-}\right)},} \\
\sup _{k \geq 1}\left\|\int_{\mathbb{R}^{N}} f_{k}(\cdot, p) d p\right\|_{L^{\infty}(\Omega)}<+\infty, \lim _{R \rightarrow+\infty} \sup _{k \geq 1}\left\|\int_{|p| \geq R} f_{k}(\cdot, p) d p\right\|_{L^{\infty}(\Omega)}=0 .
\end{gathered}
$$

We can assume (after extraction eventually) that

$$
\begin{gathered}
f_{k} \rightarrow f \text { weakly } \star \text { in } L^{\infty}\left(\Omega \times \mathbb{R}^{N}\right), \\
\mu_{k} \rightarrow \rho:=\int_{\mathbb{R}^{N}} f(\cdot, p) d p \text { weakly } \star \text { in } L^{\infty}(\Omega), \\
\phi_{k} \rightarrow \phi \text { strongly in } H^{1}(\Omega) .
\end{gathered}
$$

By taking into account that $v_{k}(p) \rightarrow \frac{p}{m}$ uniformly on compact sets of $\mathbb{R}^{N}$ we deduce easily that $(f, \phi)$ is a weak solution for the stationary Vlasov-Poisson system (11), (12), (13). Moreover the function $f$ satisfies

$$
\int_{\mathbb{R}^{N}} f(\cdot, p) d p \in L^{\infty}(\Omega), \lim _{R \rightarrow+\infty}\left\|\int_{|p| \geq R} f(\cdot, p) d p\right\|_{L^{\infty}(\Omega)}=0 .
$$




\section{References}

[1] N. B. Abdallah, Weak solutions of the initial-boundary value problem for the VlasovPoisson system, Math. Methods Appl. Sci. 17 (1994), no. 6, 451-476.

[2] H. Andréasson, The Einstein-Vlasov system/kinetic theory, Living Rev. Relativ. 5 (2002), 2002-7, 33 pp.

[3] A. Arsen'ev, Global existence of a weak solution of the Vlasov system of equations, U.R.S.S. Comp. and Math. Phys. 15 (1975), 131-143.

[4] C. Bardos, Problèmes aux limites pour les équations aux dérivees partielles du premier ordre à coefficients réels; théoremès d'approximation; application à l'équation de transport, Ann. Sci. École Norm. Sup. (4) 3 (1970), 185-233.

[5] J. Batt, W. Faltenbacher, and E. Horst, Stationary spherically symmetric models in stellar dynamics, Arch. Rational Mech. Anal. 93 (1986), no. 2, 159-183.

[6] J. Batt, P. Morrison, and G. Rein, Linear stability of stationary solutions of the VlasovPoisson system in three dimensions, Arch. Rational Mech. Anal. 130 (1995), no. 2, $163-182$.

[7] M. Bostan, Boundary value problem for the three dimensional time periodic VlasovMaxwell system, Commun. Math. Sci. 3 (2005), no. 4, 621-663.

[8] _ _ Asymptotic behavior of weak solutions for the relativistic Vlasov-Maxwell equations with large light speed, J. Differential Equations 227 (2006), no. 2, 444-498.

[9] _ Stationary solutions for the one dimensional Nordström-Vlasov system, Preprint 22 (2006), Université de Franche-Comté.

[10] F. Bouchut, F. Golse, and C. Pallard, Classical solutions and the Glassey-Strauss theorem for the $3 D$ Vlasov-Maxwell system, Arch. Ration. Mech. Anal. 170 (2003), no. 1, $1-15$.

[11] S. Calogero, Spherically symmetric steady states of galactic dynamics in scalar gravity, Classical Quantum Gravity 20 (2003), no. 9, 1729-1741.

[12] S. Calogero and H. Lee, The non-relativistic limit of the Nordström-Vlasov system, Commun. Math. Sci. 2 (2004), no. 1, 19-34.

[13] S. Calogero and G. Rein, On classical solutions of the Nordström-Vlasov system, Comm. Partial Differential Equations 28 (2003), no. 11-12, 1863-1885.

[14] Global weak solutions to the Nordström-Vlasov system, J. Differential Equations 204 (2004), no. 2, 323-338.

[15] P. Degond, Local existence of solutions of the Vlasov-Maxwell equations and convergence to the Vlasov-Poisson equations for infinite light velocity, Math. Methods Appl. Sci. 8 (1986), no. 4, 533-558.

[16] R. J. Diperna and P.-L. Lions, Global weak solutions of Vlasov-Maxwell systems, Comm. Pure Appl. Math. 42 (1989), no. 6, 729-757.

[17] _ Ordinary differential equations, transport theory and Sobolev spaces, Invent. Math. 98 (1989), no. 3, 511-547.

[18] J. Dolbeault, O. Sanchez, and J. Soler, Asymptotic behaviour for the Vlasov-Poisson system in the stellar-dynamics case, Arch. Ration. Mech. Anal. 171 (2004), no. 3, 301327.

[19] R. Glassey and J. Schaeffer, On the "one and one-half dimensional" relativistic VlasovMaxwell system, Math. Methods Appl. Sci. 13 (1990), no. 2, 169-179.

[20] _ The "two and one-half-dimensional" relativistic Vlasov Maxwell system, Comm. Math. Phys. 185 (1997), no. 2, 257-284.

[21] R. Glassey and W. Strauss, Singularity formation in a collisionless plasma could occur only at high velocities, Arch. Rational Mech. Anal. 92 (1986), no. 1, 59-90.

[22] C. Greengard and P.-A. Raviart, A boundary-value problem for the stationary VlasovPoisson equations: the plane diode, Comm. Pure Appl. Math. 43 (1990), no. 4, 473-507.

[23] Y. Guo, Global weak solutions of the Vlasov-Maxwell system with boundary conditions, Comm. Math. Phys. 154 (1993), no. 2, 245-263. 
[24] S. Klainerman and G. Staffilani, A new approach to study the Vlasov-Maxwell system, Commun. Pure Appl. Anal. 1 (2002), no. 1, 103-125.

[25] H. Lee, The classical limit of the relativistic Vlasov-Maxwell system in two space dimensions, Math. Methods Appl. Sci. 27 (2004), no. 3, 249-287.

[26] P.-L. Lions and B. Perthame, Propagation of moments and regularity for the 3dimensional Vlasov-Poisson system, Invent. Math. 105 (1991), no. 2, 415-430.

[27] S. Mischler, On the trace problem for solutions of the Vlasov equation, Comm. Partial Differential Equations 25 (2000), no. 7-8, 1415-1443.

[28] G. Nordström, Zur Theorie der Gravitation vom Standpunkt des Relativitätsprinzips, Ann. Phys. 347 (1913), no. 13, 533-554.

[29] K. Pfaffelmoser, Global classical solutions of the Vlasov-Poisson system in three dimensions for general initial data, J. Differential Equations 95 (1992), no. 2, 281-303.

[30] F. Poupaud, Boundary value problems for the stationary Vlasov-Maxwell system, Forum Math. 4 (1992), no. 5, 499-527.

[31] G. Rein, Existence of stationary, collisionless plasmas in bounded domains, Math. Methods Appl. Sci. 15 (1992), no. 5, 365-374.

[32] Non-linear stability for the Vlasov-Poisson system-the energy-Casimir method, Math. Methods Appl. Sci. 17 (1994), no. 14, 1129-1140.

[33] _ Selfgravitating systems in Newtonian theory-the Vlasov-Poisson system, Mathematics of gravitation, Part I (Warsaw, 1996), 179-194, Banach Center Publ., 41, Part I, Polish Acad. Sci., Warsaw, 1997.

[34] _ Stationary and static stellar dynamic models with axial symmetry, Nonlinear Anal. 41 (2000), no. 3-4, Ser. A: Theory Methods, 313-344.

[35] G. Rein and A. D. Rendall, Global existence of classical solutions to the Vlasov-Poisson system in a three-dimensional, cosmological setting, Arch. Rational Mech. Anal. 126 (1994), no. 2, 183-201.

[36] A. D. Rendall, An introduction to the Einstein-Vlasov system, Mathematics of gravitation, Part I (Warsaw, 1996), 35-68, Banach Center Publ., 41, Part I, Polish Acad. Sci., Warsaw, 1997.

[37] _ The Einstein-Vlasov system, The Einstein equations and the large scale behavior of gravitational fields, 231-250, Birkhauser, Basel, 2004.

[38] J. Schaeffer, The classical limit of the relativistic Vlasov-Maxwell system, Comm. Math. Phys. 104 (1986), no. 3, 403-421.

[39] Global existence of smooth solutions to the Vlasov-Poisson system in three dimensions, Comm. Partial Differential Equations 16 (1991), no. 8-9, 1313-1335.

Laboratoire de Mathématiques de Besançon

UMR CNRS 6623, Université de Franche-Comté

16 route de Gray, 25030 Besançon Cedex France

E-mail address: mbostan@univ-fcomte.fr 